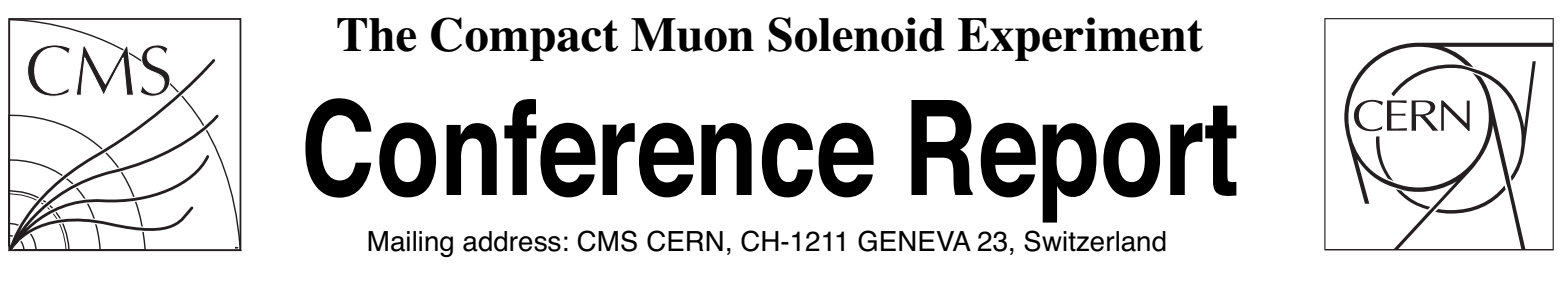

19 April 2020 (v5, 30 May 2020)

\title{
Production and installation of the first GEM station in CMS
}

\author{
Simone Calzaferri on behalf of the CMS Collaboration
}

\begin{abstract}
In 2017 and during the whole $2018 \mathrm{LHC}$ has reached the record beam luminosity $2 \cdot 10^{34} \mathrm{~cm}^{-2} \mathrm{~s}^{-1}$, around a factor of two beyond the LHC design. In December 2018 the Large Hadron Collider (LHC) entered the LS2 phase (Long Shutdown 2), which will last until the beginning of 2021, in which the maintenance program of LHC and the other smaller accelerators is scheduled.

To cope with this and also looking at the following LHC phase in which the luminosity will be increased to $5 \cdot 10^{34} \mathrm{~cm}^{-2} \mathrm{~s}^{-1}$ the LHC experiments must be upgraded as well. In this context the muon subsystem of the CMS experiment began installing the first GEM based detectors station (GE1/1) in July 2019, almost 5 meters from the point of interaction.

GE1/1 will consist of 144 triple Gas Electron Multiplier (GEM) detectors, which will work together with the Cathode Strip Chamber (CSC) station ME1/1, improving tracking and triggering of muons produced in the pseudorapidity in range $1.6<|\eta|<2.15$.

The installation in CMS is now underway at a good pace. The first 72 chambers have been installed together with their services (gas, cooling, low voltage and high voltage etc.) while the completion of the station is foreseen in spring 2020. This contribution will summarize the detector design along with the steps a detector must pass before being approved for the installation in CMS. The status of the operations will be presented, together with the tools developed to monitor the detector parameters during installation. Finally the plans for the installation and commissioning of the entire project will be outlined.
\end{abstract}




\title{
Production and installation of the first GEM station in CMS
}

\author{
S. Calzaferri, ${ }^{a, b, 1}$ on behalf of the CMS collaboration \\ ${ }^{a}$ Università degli studi di Pavia, via A. Bassi 6, Pavia, Italy \\ ${ }^{b}$ INFN sezione di Pavia, via A. Bassi 6, Pavia, Italy \\ E-mail: simone.calzaferri@cern.ch
}

AbSTRACT: In 2017 the LHC reached the record beam luminosity $2 \cdot 10^{34} \mathrm{~cm}^{-2} \mathrm{~s}^{-1}$, around a factor of two beyond the LHC design value. In December 2018 the Large Hadron Collider (LHC) entered the LS2 phase (Long Shutdown 2), which will last until beginning of 2021, in which the maintainance and upgrade program of LHC and the other smaller accelerators is scheduled.

To cope with this and also looking at the following LHC phase in which the luminosity will be increased to $5 \cdot 10^{34} \mathrm{~cm}^{-2} \mathrm{~s}^{-1}$ the LHC experiments must be upgraded as well. In this context the muon subsystem of the CMS experiment began installing the first GEM based detector station (GE1/1) in July 2019, almost 5 meters from the point of interaction.

GE1/1 will consist of 144 triple Gas Electron Multiplier (GEM) detectors, which will work together with the Cathode Strip Chamber (CSC) station ME1/1, improving tracking and triggering of muons produced in the pseudorapidity range of $1.6<|\eta|<2.15$.

The installation in CMS is now underway at a good pace. The first 72 chambers have been installed together with their services (gas, cooling, low voltage and high voltage etc.) while the completion of the station is foreseen in spring 2020. This contribution will summarize the detector design along with the steps a detector must pass before being approved for the installation in CMS. The status of the operations will be presented, together with the tools developed to monitor the detector parameters during installation. Finally the plans for the installation and commissioning of the entire project will be outlined.

KEYWORDS: GEM detectors, CMS upgrade

\footnotetext{
${ }^{1}$ Corresponding author.
} 


\section{Contents}

1 Introduction 1

2 GE1/1 detector production and quality control 1

3 Status of GE1/1 installation in CMS 3

$4 \quad$ HV and LV monitor tool $\quad 4$

5 Conclusion 5

\section{Introduction}

In LHC Run 2, CMS has used three different technologies for muon tracking and triggering:

- Drift Tubes (DTs),

- Resistive Plate Chambers (RPCs),

- Cathode Strip Chambers (CSCs).

From LHC Run 3 (scheduled for 2021), CMS will introduce Gas Electron Multiplier detectors for muon tracking and triggering. GEM technology has been tested in CMS in 2016 during the LHC Run 2 for gaining an operational experience and measure the muon detection efficiencies and background rates. This experience has been named GEM Slice Test [4].

In CMS, GEM detectors will be installed in three stations:

- GE1/1 $(1.6<|\eta|<2.15)$,

- GE2/1 $(1.6<|\eta|<2.4)$,

- ME0 $(2.0<|\eta|<2.8)$, increasing the CMS $\eta$-coverage for muon detection.

This technology was selected because of the high rate capability (up to $100 \mathrm{MHz} \mathrm{cm}^{-2}$ ), the high spatial resolution $\left(140 \mu \mathrm{m}\right.$ ) and the high radiation hardness (up to $1.56 \mathrm{C} \mathrm{cm}^{-2}$ ). Furthermore, GEMs will increase the redundancy and provide a higher number of hits to reconstruct muons. The GE1/1 and GE2/1 stations will help to keep the trigger rate under control without increasing the muon $p_{T}$ threshold. Three stations are foreseen to be installed by the end of LHC Long Shutdown 3 (LS3).

\section{GE1/1 detector production and quality control}

GE1/1 is made of 144 GEM detectors, each of them consisting of three GEM foils stacked one over the other. A GEM foil is a $50 \mu \mathrm{m}$ thick polymer foil, with copper on both sides, with $70 \mu \mathrm{m}$ biconical holes (etched on foils) interspaced by $140 \mu \mathrm{m}$. During its operation the GEM detector is filled with a gas mixture $\left(\mathrm{Ar} / \mathrm{CO}_{2} 70 \% / 30 \%\right)$. An $\mathrm{HV}$ multichannel power supply creates a high electric field between the cathode and the anode planes, respectively, at the top and bottom 


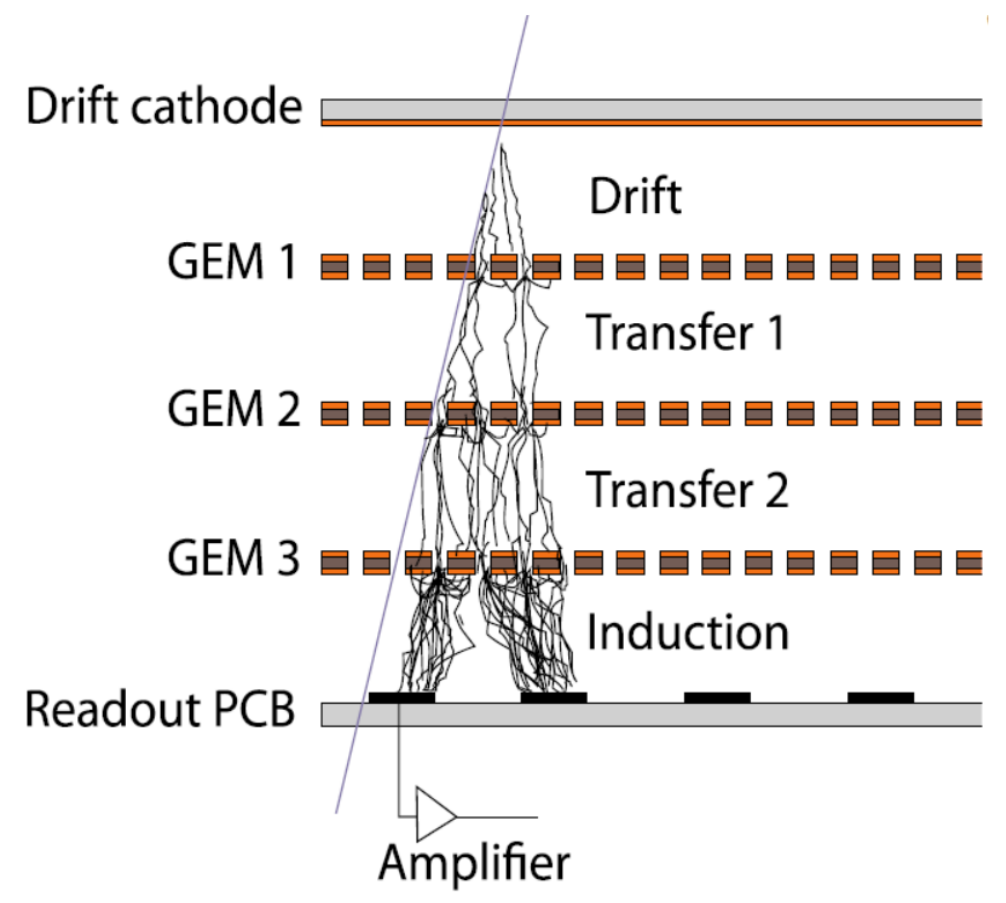

Figure 1. Structure of a GE1/1 detector [2].

as shown in Fig. 1. In particular the electric field in holes $(\sim 80 \mathrm{kV} / \mathrm{cm})$ is stronger than in gaps $(\sim 3 \mathrm{kV} / \mathrm{cm})$.

When a particle enters in the detector gas medium it produces ionisation (electrons and ions) by the effect of electric field. The electrons drift towards the anode while the ions drift in the opposite direction. When electrons pass inside the GEM foil holes, an avalanche multiplication is produced, by the stronger electric field in the holes, and they are finally collected on the anode strips, where their signal is read with a custom designed electronics.

The production of the 144 GE1/1 detectors is shared among six labs around the world. The chamber assembly consists in two steps: the preparation of materials in the lab and the assembly of GEM detector in a clean room, to avoid that any kind of dust enters in the detector and cause any issues in the detector operation.. In the preparation the needed components are cleaned, the HV circuit is prepared, pull-outs are mounted and the O-rings needed for the assembly are selected. In the assembly phase in the clean room a fast test of GEM folis is performed, the stack of GEM foils is mounted and finally the GEM detector is closed. The assembly procedure of a GEM detector takes approximatively one day.

During the production and validation of GEM detectors several quality controls and operations are performed:

- GEM foils test to check for undesirable short circuits,

- gas leak test to check for chamber gas tightness,

- HV circuit test,

- gas gain calibration to determine the chamber operation gain,

- HV stability test to determine the chamber trip rate and clean the foils after a long inactivity 
period,

- electronics connectivity test and cooling circuit mounting.

When all these operations have been checked thoroughly two GE1/1 chambers are coupled as a super-chamber, resulting in a total number of 72 super-chambers, 36 per each CMS endcap. The super-chamber muon detection efficiency is tested in a specially designed cosmic stand. A schematic representation of production and validation of detectors is reported in Fig. 2.

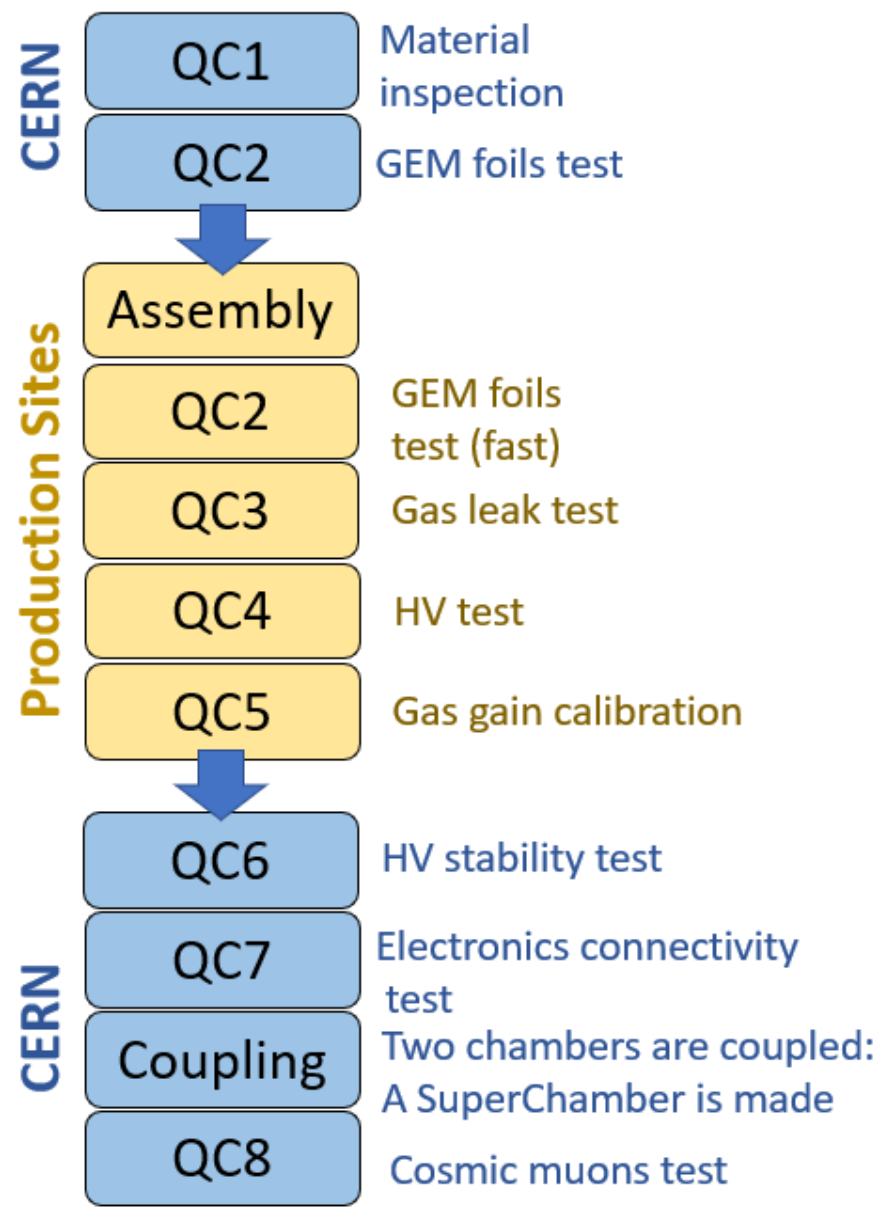

Figure 2. Schematic representation of production and validation of GE1/1 detectors

\section{Status of GE1/1 installation in CMS}

The installation of GE1/1 in CMS was started in July 2019 and is now progressing well. Once a GE1/1 super-chamber has passed all the quality controls it is brought to the CMS experiment site and lowered to the CMS experimental cavern though the shaft connecting the cavern to the surface. The super-chamber is the mounted in the installation jig and installed in its dedicated site (Fig. 3).

In the CMS negative endcap all 36 GE1/1 super-chambers are installed together with their services such as gas and cooling, HV and LV systems. The GEM Detector Control System (DCS) 


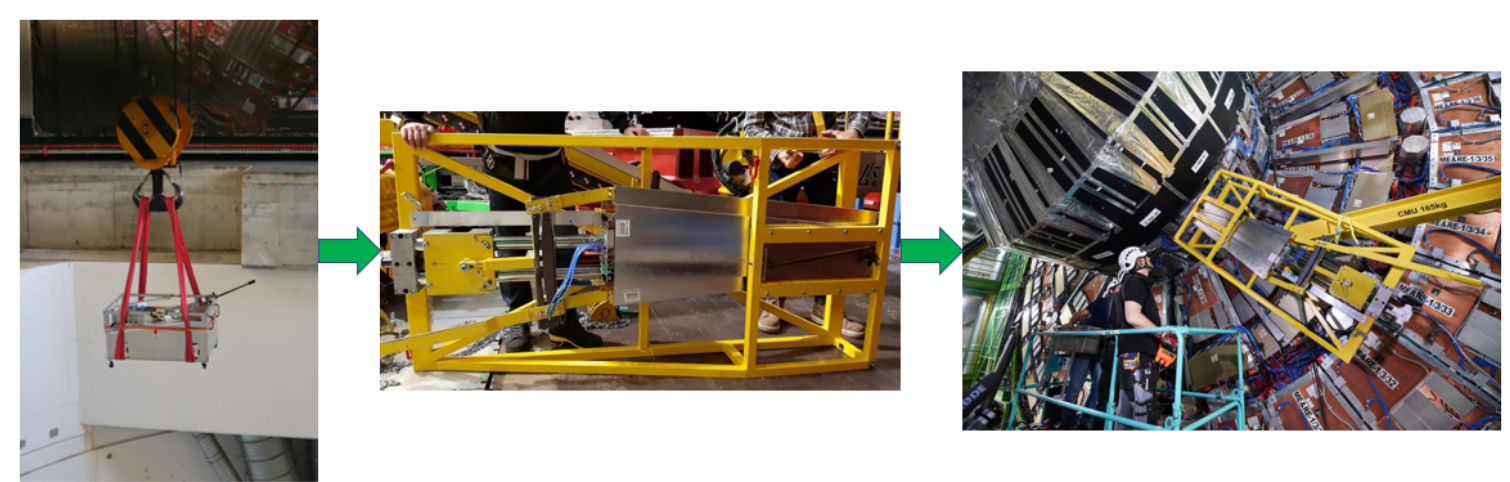

Figure 3. Installation steps of a GE1/1 super-chamber in the CMS detector: the lowering to the CMS experimental cavern (left), the mounting in the installation jig (middle), the installation in CMS detector

is under local test and the readout back-end hardware has been installed. On the other hand, in the positive endcap super-chambers will be installed in the next few months. The positive endcap systems services (gas, cooling, HV, LV, DAQ electronics and fibers) are under installation. The detailed status of the installation of every GE1/1 chamber in the CMS negative endcap can be figured out from Fig. 4.
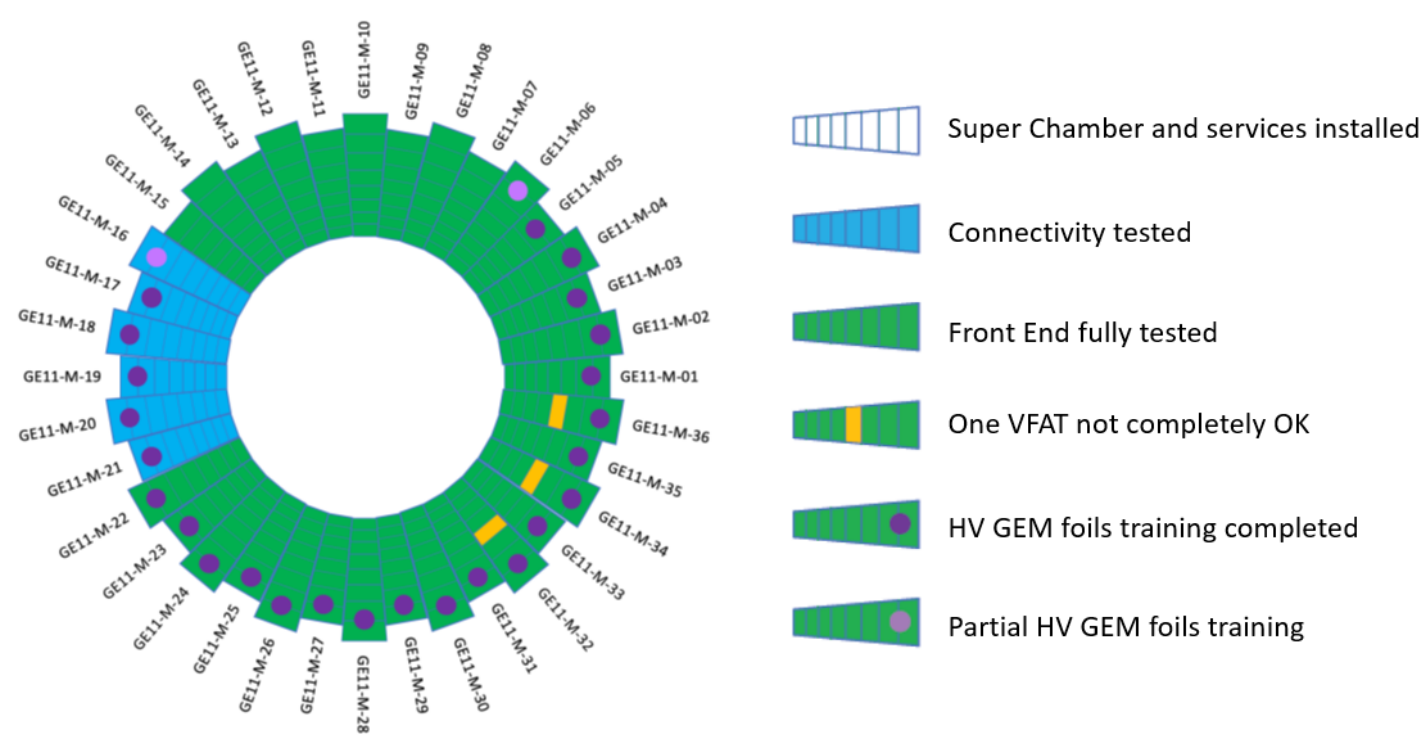

Figure 4. Detailed status of the installation of GE1/1 chambers in the CMS negative endcap

\section{$4 \quad$ HV and LV monitor tool}

Every time a GEM chamber is installed in CMS, the HV stability of GEM foils and the communication with its front-end electronics is checked. To perform these tests, HV and LV are powered by the DCS, which stores every applied HV and LV value in a database. During an operation period and the detector commissioning some problems may arise (e.g. a HV or LV board can operate 
incorrectly or a chamber can start tripping). One possible way to inspect the problem is checking the $\mathrm{HV}$ and LV values stored in the CMS database for the problematic chamber for which a scanning tool has been developed to retrieve and debug. Fig. 5 shows in particular the current and voltage
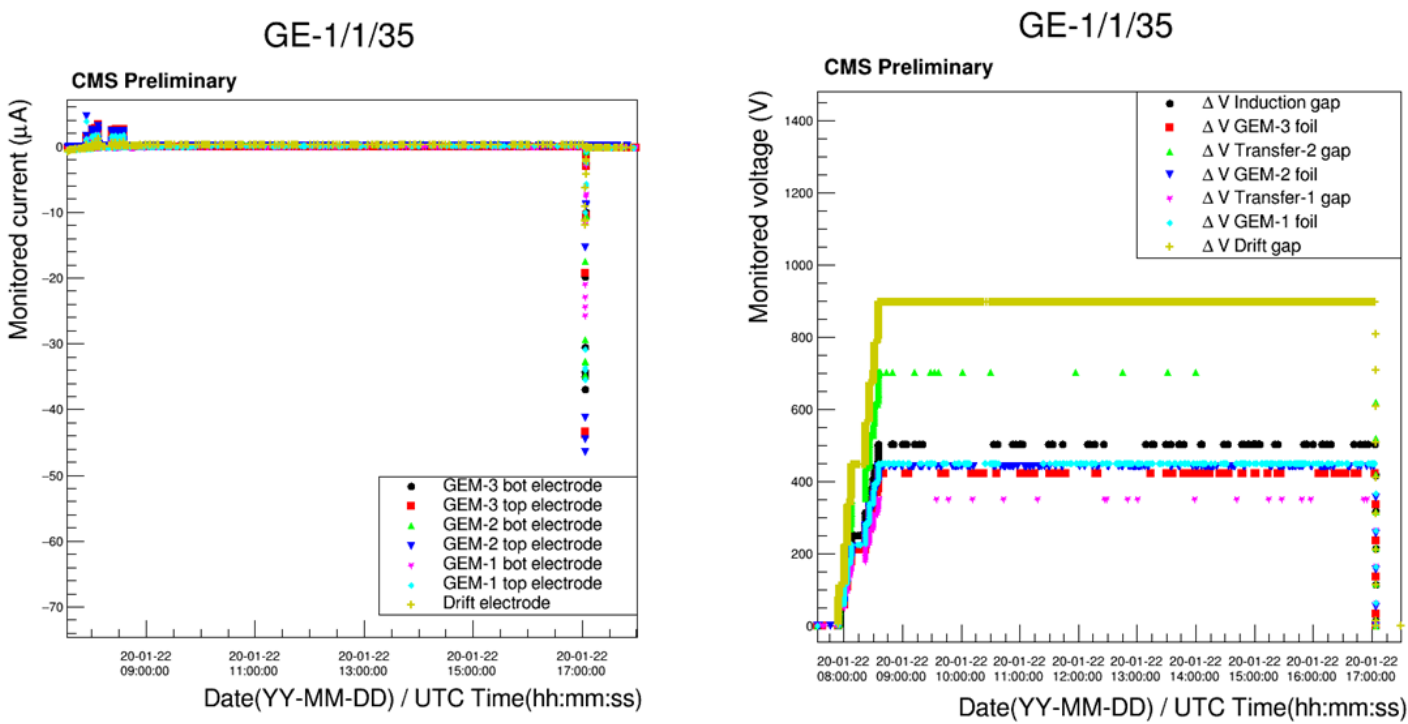

Figure 5. On the left, current flowing on top or bottom faces of GEM foils and on the cathode of GE1/1 chamber 35 in the negative endcap of CMS during the GEM foil training [3]; on the right, voltage differences created in gas gaps or between the top and bottom part of one GEM foil of GE1/1 chamber 35 in the negative endcap of CMS during the GEM foil training [3].

differences applied on the electrodes powering the GE1/1 chamber 35 placed in the CMS negative endcap registered during the GEM foil training after the chamber installation. It can be observed that voltage and current stay stable on all the 7 electrodes for the whole duration of the GEM foils training (almost 9 hours) and variation in current is observed only in the initial and final phases, when the voltage is varied to power the detector on or off.

\section{Conclusion}

The validation and installation of GE1/1 chambers in CMS is ongoing and will be completed before the LHC Run 3. For the commissioning of chambers the tool developed to check the status of the power system for GE1/1 chambers is particularly useful, as it can be used to spot and potentially solve issues that may arise during chamber operation.

\section{References}

[1] CMS Collaboration, The CMS experiment at the CERN LHC, JINST $3 \mathbf{S 0 8 0 0 4}$ (2008)

[2] CMS Collaboration, The Phase-2 Upgrade of the CMS Muon Detectors, Technical Design Report, CERN-LHCC-2017-012 CMS-TDR-016 (12 September 2017)

[3] GEM DPG Public Page, https://twiki . cern. ch/twiki/bin/view/CMSPublic/GEMDPGPublic 
[4] CMS Collaboration, Commissioning and performance of the GE1/1 slice test detectors, CERN Document Server, http://cds. cern.ch/record/2629838?ln=it, CMS-CR-2018-071 (7 June 2018) 